\title{
Nucleation and lateral growth kinetics of the NiSi phase at the epitaxial $\theta-\mathrm{Ni}_{2} \mathrm{Si} / \mathrm{Si}$ interface
}

\author{
M. El Kousseifi ${ }^{1}$, K. Hoummada ${ }^{1}$, F. Panciera ${ }^{1, \dagger}$, C. Lavoie $^{2}$, D. Mangelinck ${ }^{1 *}$ \\ ${ }^{1}$ IM2NP, Aix Marseille Université-CNRS, UMR 7334, Faculté de Saint Jérôme, 13397 Marseille, France \\ ${ }^{2}$ IBM Thomas J. Watson Research Center, Yorktown Heights, New York 10598, USA \\ †Current address: Université Paris-Saclay, CNRS, Centre de Nanosciences et de Nanotechnologies, 91120, Palaiseau,
} France.

\begin{abstract}
The first stages of the growth of the NiSi phase at the expense of $\theta-N i_{2}$ Si have been studied mainly by in-situ XRD measurements and atom probe tomography (APT) analysis. In-situ XRD isothermal annealing at different temperatures were performed on several samples in order to monitor the phase formation sequence, the time at which NiSi phase begin to form and its growth kinetics. These results show that while the phase formation sequence is the same, the time for the beginning of formation of NiSi varies from one sample to the other under the same isothermal temperature and experimental conditions. Comparing these findings with nucleation and growth models, the growth of the NiSi phase at the expense of $\theta-\mathrm{Ni}_{2} \mathrm{Si}$ is controlled by nucleation compared to diffusion in the case of $\delta$-Ni $\mathrm{N}_{2} \mathrm{Si}$ as the first phase. The kinetics for the nucleation and lateral growth of the NiSi phase were deduced and the implications for the formation of this phase and for contacts are discussed.
\end{abstract}

Keywords: Nucleation; Thin film; Growth kinetics; In situ x ray diffraction; Nickel silicide

* Corresponding author 


\section{Introduction}

Ni-based self-aligned silicides are widely used as contacts and interconnections in ultra-large-scale integrated circuits [1]. They are obtained by a solid-state reaction between a Ni thin film and a $\mathrm{Si}$ substrate. This reaction leads usually to the formation of $\delta-\mathrm{Ni}_{2} \mathrm{Si}$ as the first phase followed by NiSi after the complete consummation of $\mathrm{Ni}$, and $\mathrm{NiSi}_{2}$ phase at higher temperature [2]. Among these, NiSi is the phase of interest for its low resistivity as a contact material for CMOS devices. Despite its advantages, NiSi suffers from severe agglomeration above $650^{\circ} \mathrm{C}$ and it transforms into the more resistive $\mathrm{NiSi}_{2}$ phase above $800^{\circ} \mathrm{C}$ [3]. It has been demonstrated that the addition of a small amount of alloying element to Ni film, such as $\mathrm{Pt}, \mathrm{Pd}$, Mo or Re, reduces the agglomeration of NiSi and prevents its transformation into $\mathrm{NiSi}_{2}[4,5]$. Therefore the addition of 5 at.\% $\mathrm{Pt}$ was first used and the $\mathrm{Pt}$ concentration has been increased in the $\mathrm{Ni}$ film from 5 at. $\%$ to 10 at. $\%$ for applications in microelectronics [6-8]. The addition of alloy elements can modify the phase sequence and in particular it has been observed that during the reaction of $\mathrm{Ni}\left(10\right.$ at.\% Pt) with $\mathrm{Si}(100), \theta-\mathrm{Ni}_{2} \mathrm{Si}$ replaces $\delta-\mathrm{Ni}_{2} \mathrm{Si}$ as first phase [9]. In this case $\theta-\mathrm{Ni}_{2} \mathrm{Si}$ grows in epitaxy with the $\mathrm{Si}$ substrate prior to the formation of $\mathrm{NiSi}[10]$. The composition of the intermixing layer formed at interface during deposition was found to control the nature of the first phase and to lead either to $\delta-\mathrm{Ni}_{2} \mathrm{Si}$ or $\theta-\mathrm{Ni}_{2} \mathrm{Si}$ [11]. The growth of NiSi at the expense of the $\delta-\mathrm{Ni}_{2} \mathrm{Si}$ phase has been extensively studied with results showing that the NiSi growth is controlled by diffusion of $\mathrm{Ni}$ as the fastest diffusion species [12-14]. In addition, it was shown that there is a time delay between the formation of the NiSi phase and the end of the growth of $\delta-\mathrm{Ni}_{2} \mathrm{Si}$ that was attributed to the strain relaxation in $\delta-\mathrm{Ni}_{2} \mathrm{Si}$ phase. Furthermore, it was demonstrated that this time delay follows Arrhenius behaviour with activation energy around 1.2 and $1.7 \mathrm{eV}$ for pure $\mathrm{Ni}$ and $\mathrm{Ni}(5$ at.\% $\mathrm{Pt})$ respectively [15]. However, the growth of $\mathrm{NiSi}$ at the expense of $\theta-\mathrm{Ni}_{2} \mathrm{Si}$ and especially its first growth stages is not well understood. Indeed, in contrast to the extensive work concerning the growth of $\mathrm{NiSi}$ at the expense of $\delta-\mathrm{Ni}_{2} \mathrm{Si}$, only a few studies have been conducted to understand the growth of $\mathrm{NiSi}$ at the expense of $\theta-\mathrm{Ni}_{2} \mathrm{Si}$. Recently, the lateral growth of $\mathrm{NiSi}$ at the 
epitaxial $\theta-\mathrm{Ni}_{2} \mathrm{Si} / \mathrm{Si}$ interface was evidenced by TEM [16]. The experimental shape of isolated NiSi precipitates resulting from the lateral growth of $\mathrm{NiSi}$ at the $\theta-\mathrm{Ni}_{2} \mathrm{Si} / \mathrm{Si}$ interface was in accordance with several models $[16,17]$.

In this work, the nucleation and the lateral growth kinetics of NiSi phase at the epitaxial $\theta-\mathrm{Ni}_{2} \mathrm{Si} / \mathrm{Si}$ interface have been investigated using in situ X-ray diffraction (XRD) and atom probe tomography (APT) measurements. Several isothermal annealing (in-situ XRD) coupling with models have been used in order to calculate the activation energy for NiSi nucleation and lateral growth kinetics.

\section{Experimental}

10-nm-thick Ni (10 at.\% Pt) films were deposited by magnetron sputtering on (100) Si substrates. The (100) Si substrates were cleaned with diluted HF prior to loading into the sputtering chamber. The deposition was performed in a sputtering deposition setup with a base pressure of $10^{-8}$ Torr using a 99.99\% pure Ar gas flow and a $99.99 \% \mathrm{Ni}(\mathrm{Pt})$ target. The wafer was rotated during the deposition to improve the homogeneity of the layer. Then, the reaction between the $\mathrm{Ni}(\mathrm{Pt})$ film and the substrate was followed by in situ XRD. For XRD measurements, a $\mathrm{Cu}$ tube and a $\theta-\theta$ geometry were used in a a Panalytical X-Pert Pro MPD diffractometer. The samples were loaded into the XRD chamber (Anton Paar TTK450), which is equipped with a heating stage and maintained under an ambient vacuum of approximately $10^{-5}$ mbar. In the XRD chamber, the sample was heeld by two stainless steel plates on a metal block sitting on a heather and inside which a thermocouple was embedded. In situ isothermal XRD analyses were performed by increasing the temperature from room temperature to the chosen temperature at a rate of $30^{\circ} \mathrm{C} / \mathrm{min}$ and by continuously recording the XRD spectra at this temperature for a given time.

TEM measurements (TITAN microscope equipped with an image Cs-corrector and operated at $300 \mathrm{kV}$ ) were performed by cross-sectional views on samples prepared by a focused ion beam dual beam instrument (FIB, FEI Helios600 NanoLab) along the direction (110) of Si after deposition of a protection layer 
The APT specimen preparation was performed using a Helios dual-beam Focused ion beam (FIB) equipped with a micromanipulator and with a gas injection system (GIS) for Pt deposition. Several steps are needed for the APT specimen preparation by FIB as described elsewhere $[18,19]$. The APT analyses were carried out in a LEAP 3000X HR instrument. The laser pulsing rate was set at $100 \mathrm{kHz}$ and the detection rate was kept at 0.002 event/pulse by increasing the applied voltage. The samples were analyzed under the following conditions: pressure in the range of $10^{-11}$ Torr, temperature of 25 $\mathrm{K}$ and laser energy of $0.5 \mathrm{~nJ}$. These analysis conditions were applied to all the analyzed samples.

\section{Results}

Figure 1.a presents the in situ XRD measurement (intensity as a function of diffraction angle and time) showing the phase formation sequence during an isothermal annealing at $215^{\circ} \mathrm{C}$ for the $10 \mathrm{~nm} \mathrm{Ni}(10$ at.\% Pt)/Si (100) sample. It can be summarized by the following. The consumption of the $\mathrm{Ni}(10$ at.\% Pt) film (XRD peak at $44^{\circ}$ ) is associated to the formation of the $\theta-\mathrm{Ni}_{2} \mathrm{Si}$ phase. $\theta-\mathrm{Ni}_{2} \mathrm{Si}$ grows in epitaxy with the Si substrate, which explains the absence of XRD peaks until the detection of NiSi phase [10]. Then, the formation of the textured NiSi phase (200) at the expense of $\theta-\mathrm{Ni}_{2} \mathrm{Si}$ is observed with a single $\mathrm{XRD}$ peak at $56.2^{\circ}$. From the in situ XRD data, the variation of the normalized integrated intensities with time are reported for Ni consumption and NiSi formation in Figure 1.b ( $1^{\text {st }}$ annealing). This experiment was repeated several times under the same conditions (as deposited sample, atmosphere and temperature) and the results are presented in Figure 1.b. The integrated intensity of a XRD peak can be considered to be proportional to the volume of the corresponding phase in the absence of change in the texture of the phase.

In all experiments at the nominal temperature of $215^{\circ} \mathrm{C}$, the time for the complete Ni consumption (i.e. the formation of $\theta-\mathrm{Ni}_{2} \mathrm{Si}$ ) was found to be reproducible within a range of 50 min while the beginning of NiSi formation changes for each annealing and varies between 188 and 366 min. However, once 
the NiSi phase is detected, the time to complete the NiSi growth is roughly the same showing that the formation kinetics is roughly similar at the nominal temperature of $215^{\circ} \mathrm{C}$.
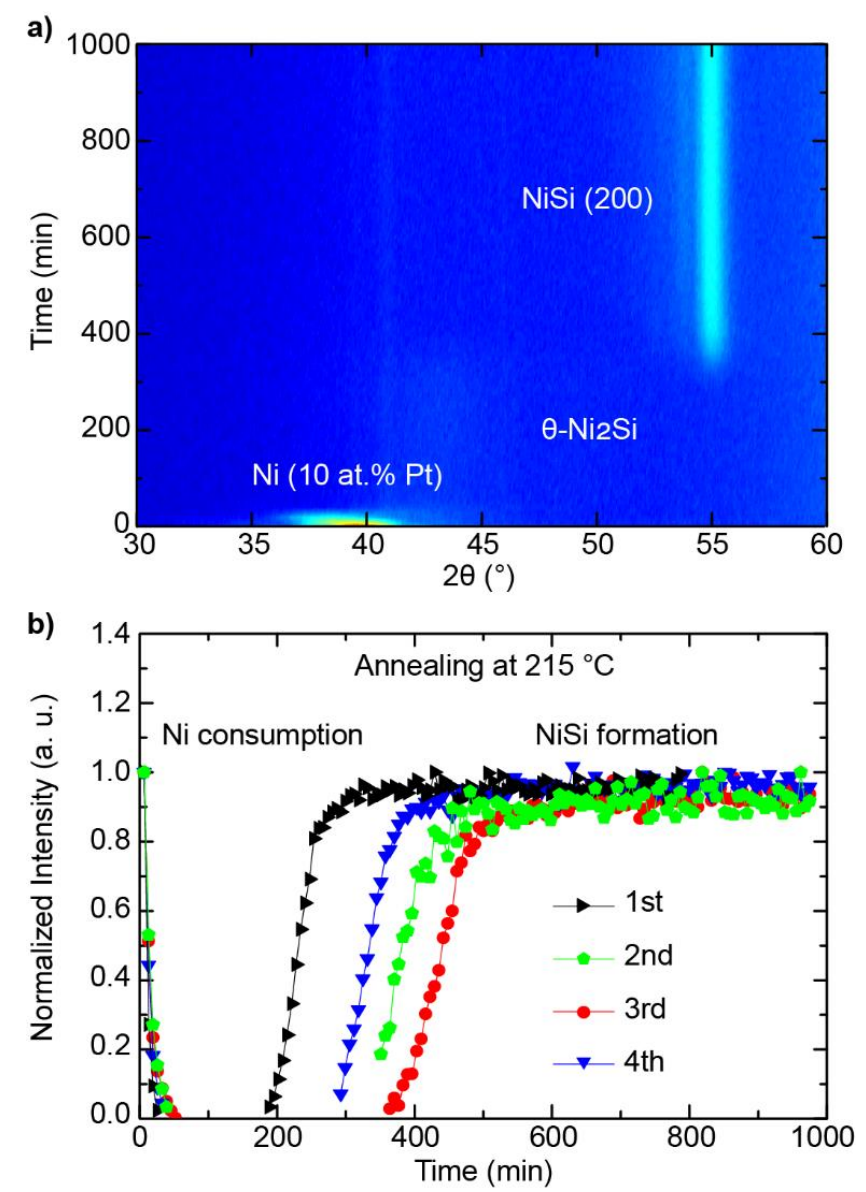

Figure 1: In-situ XRD diagram $(\lambda=1.54 \AA)$ during isothermal annealing at $215{ }^{\circ} \mathrm{C}$ on $10 \mathrm{~nm} \mathrm{Ni}(10$ at.\% $\mathrm{Pt}) / \mathrm{Si}(100)$ sample. (a) XRD intensities as a function of diffraction angle measured during the heat treatment (b) Variation of the normalized integrated intensity of the XRD peaks as a function of time during four isothermal annealing at the same nominal temperature $\left(215^{\circ} \mathrm{C}\right)$.

Isothermal experiments at other temperatures $\left(180,200,230\right.$, and $\left.250{ }^{\circ} \mathrm{C}\right)$ have also been performed in order to deduce the growth kinetic and to shed light on the variability of the start of NiSi formation. Figure 2 shows the normalized integrated intensities of XRD peaks as a function of time for the Ni consumption and NiSi formation during several isothermal annealing at 200 and $230{ }^{\circ} \mathrm{C}$. The Ni consumption is similar for a given temperature and becomes faster with increasing temperature, around 
$100 \mathrm{~min}$ at $200{ }^{\circ} \mathrm{C}, 50 \mathrm{~min}$ at $215^{\circ} \mathrm{C}$ and $15 \mathrm{~min}$ at $230{ }^{\circ} \mathrm{C}$. The beginning of the NiSi formation appears to occur at different times for each isothermal annealing. The difference between the end of $\mathrm{Ni}$ consumption and the beginning of NiSi formation will be now defined as the time delay for NiSi formation. For example, at $230{ }^{\circ} \mathrm{C}$, the time delay for NiSi formation varies between 2 and 75 min.
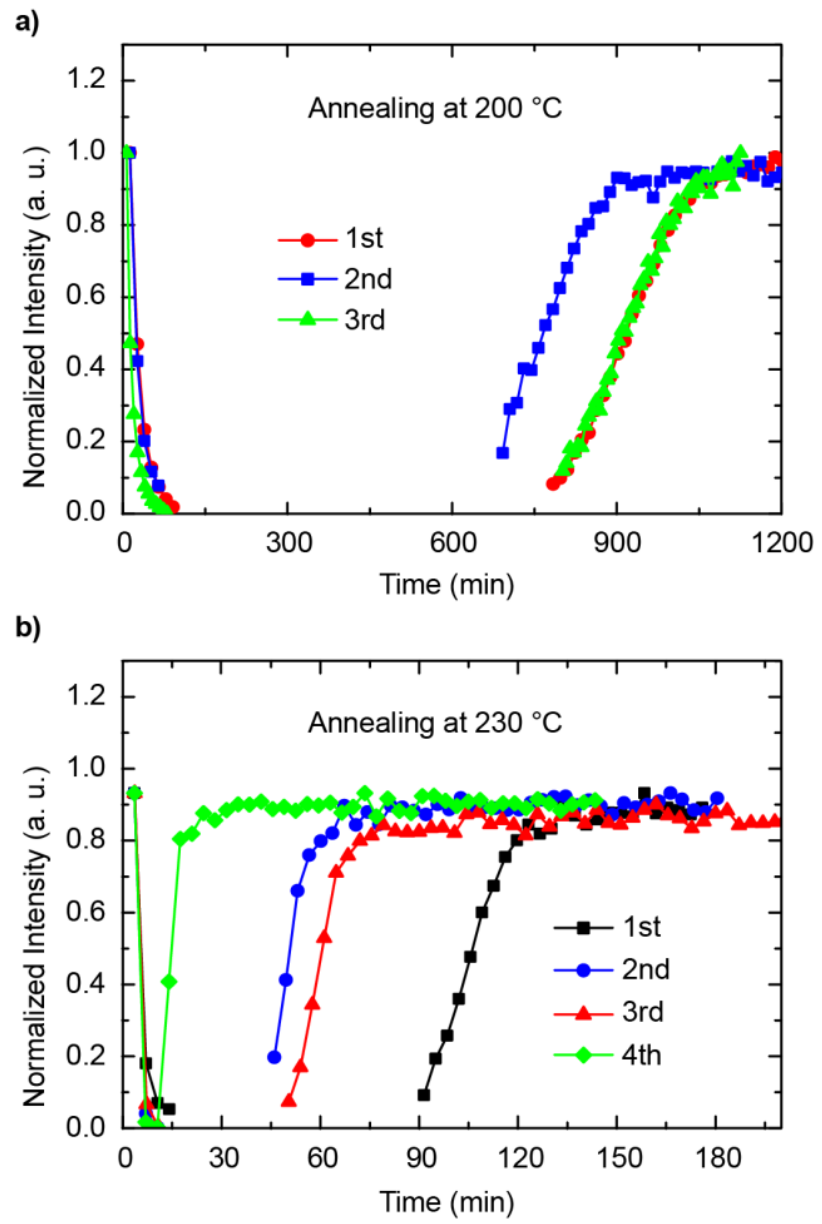

Figure 2: Results obtained by in-situ XRD diagram $(\lambda=1.54 \AA)$ during two isothermal annealing on $10 \mathrm{~nm} \mathrm{Ni(10} \mathrm{at. \%}$ $\mathrm{Pt}) / \mathrm{Si}(100)$ sample. The variation of the normalized intensity of the XRD peaks as a function of time after two isothermal annealing a) at $200{ }^{\circ} \mathrm{C}$ and b) $230{ }^{\circ} \mathrm{C}$.

From these results, the average time delay has been estimated for each temperature. This average time delay decreases with temperature $\left(\sim 30 \mathrm{~min}\right.$ for $230{ }^{\circ} \mathrm{C}, 150 \mathrm{~min}$ for $215^{\circ} \mathrm{C}$ and $600 \mathrm{~min}$ for $\left.200{ }^{\circ} \mathrm{C}\right)$. It appears that with the temperature increase, the slope representing the growth of NiSi becomes steeper which means that the growth kinetics has accelerated, as expected. We also notice that for a given temperature the slope of samples that nucleate earlier is steeper than samples that nucleate later. This 
suggests that the variation in nucleation time could be due to a small difference in temperature between different experiments. This hypothesis will be verified in the discussion.

In order to better understand the origin of these time delays, transmission electron microscopy (TEM) and atom probe tomography (APT) have been used to characterize the nanostructure and composition of the sample. Therefore, another in-situ XRD annealing at $215^{\circ} \mathrm{C}$ has been performed and stopped after the first appearance of the characteristic peak of NiSi at $2 \theta$ equal $56^{\circ}$. Then, TEM and APT were used after the annealing to characterize the sample. Figure 3.a is a TEM cross section showing a typical $\mathrm{NiSi}$ precipitate at the $\theta-\mathrm{Ni}_{2} \mathrm{Si} / \mathrm{Si}(100)$ interface. It was shown previously $[16,17]$ that these precipitates are due to the lateral growth of $\mathrm{NiSi}$ at the epitaxial $\theta-\mathrm{Ni}_{2} \mathrm{Si} / \mathrm{Si}(100)$ interface, with the existence of a low density of NiSi nuclei at the interface. Figure 3. b and c show two APT volumes that correspond to the analysis performed on this first stage of the NiSi growth. The analysis direction is perpendicular to the surface and the interfaces and each element $(\mathrm{Ni}, \mathrm{Si}$ and $\mathrm{Pt})$ is represented by a color. The black line in all the slices corresponds to the interface Ni silicide/Si substrate and it was marked with an isoconcentration of 70 at.\% Si. It was noted that the Ni distribution in Figure 3.b is homogeneous (Ni silicide) in the first obtained volume, which indicates the presence of one phase. The Ni silicide with a thickness of $17 \mathrm{~nm}$ shows a constant Si concentration of 40 at.\% (Figure 3.b) that is a signature of the $\theta-\mathrm{Ni}_{2} \mathrm{Si}$ phase. In contrast, observing the $\mathrm{Ni}$ and $\mathrm{Si}$ distribution in the second volume (Figure 3.c), two contrasts can be observed in the Ni silicide layer indicating the presence of two phases. The composition of these phases was obtained by APT concentration profile (Figures 3.d and 3.e) indicating the presence of $\theta-\mathrm{Ni}_{2} \mathrm{Si}$ and $\mathrm{NiSi}$ phases. This concentration profiles shows clearly the formation of $\mathrm{NiSi}$ at the epitaxial $\theta-\mathrm{Ni}_{2} \mathrm{Si} / \mathrm{Si}$ interface. During the reconstruction of the APT volumes, the total depth was calibrated using the TEM images: this results in an agreement between TEM (Figure 3.a) and APT (Figures 3d. and 3e) for the thicknesses of $\theta-\mathrm{Ni}_{2} \mathrm{Si}$ and $\mathrm{NiSi}$. The Pt redistribution during the formation of $\theta-\mathrm{Ni}_{2} \mathrm{Si}$ and $\mathrm{NiSi}$ phases is presented in Figure 3.c and 3.d. Generally, $\mathrm{Pt}$ presents an exponential profile in $\theta-\mathrm{Ni}_{2} \mathrm{Si}$ during the reaction of $\mathrm{Ni}(10 \% \mathrm{Pt})$ with $\mathrm{Si}$ and seems to be 
accumulated on the surface of the phase [10]. During the NiSi growth, the Pt accumulation is located at the interface separating the two phases indicating the low Pt diffusion in NiSi.

a)

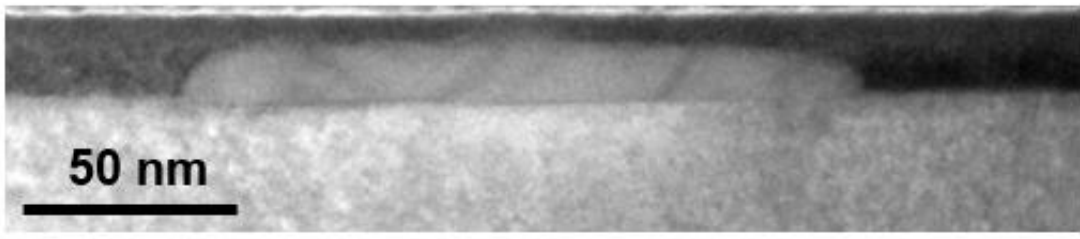

Analysis direction

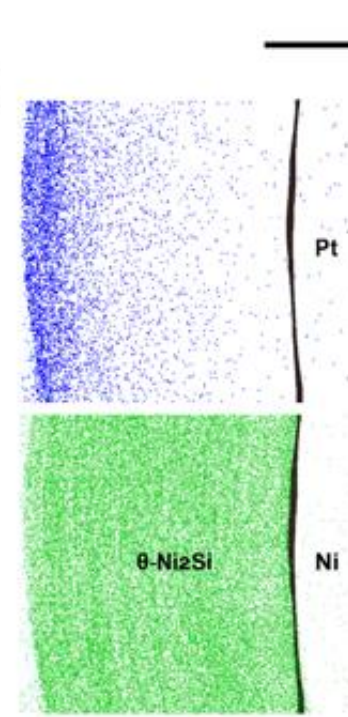

b)

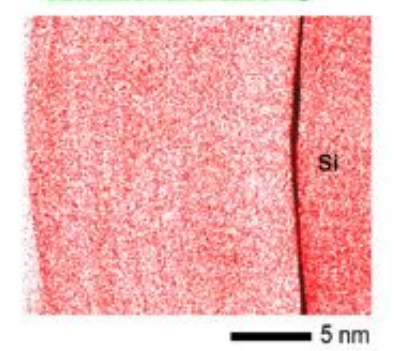

d)

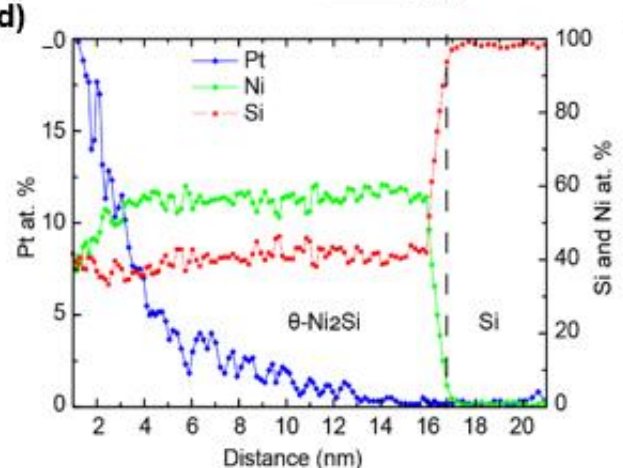

c)
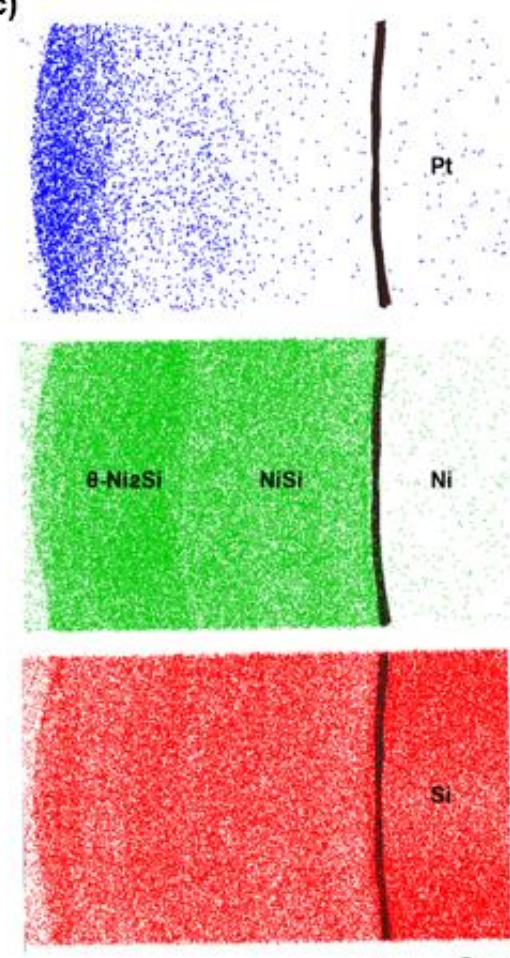

e)

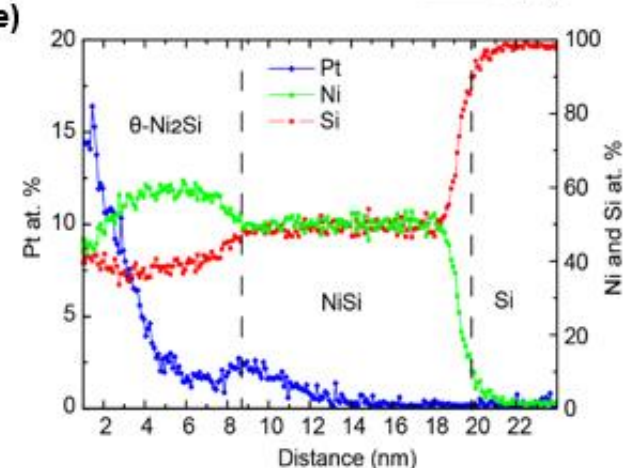

* a high resolution figure is provided as a separated file since it is very large

Figure 3: TEM and APT characterization after isothermal annealing at $215^{\circ} \mathrm{C}$ using an in-situ XRD measurement stopped after the first appearance of NiSi phase: a) TEM cross section showing a precipitate of NiSi at the epitaxial $\theta$ - 
$\mathrm{Ni}_{2} \mathrm{Si} / \mathrm{Si}(100)$ interface. b) and c) $5 \mathrm{~nm}$ thick slices of two APT volumes showing the distribution of Pt (blue dots), Ni

(green dots) and $\mathrm{Si}$ (red dots) atoms. Only $\theta-\mathrm{Ni}_{2} \mathrm{Si}$ is present in b) while NiSi is also present in c). The silicide/Si interface is shown with an iso-concentration of 70 at.\% Si. d) and e) 1D concentration profiles corresponding to the APT volume in $\mathrm{b}$ ) and c): the profile was taken along the $\mathrm{z}$ direction perpendicular to the $\theta-\mathrm{Ni}_{2} \mathrm{Si} / \mathrm{Si}$ interface.

The APT results are in accordance with the TEM results $[16,17]$ and show that the NiSi does not form a uniform layer at the interface $\theta-\mathrm{Ni}_{2} \mathrm{Si} / \mathrm{Si}$, but precipitates dispersed at this interface. Indeed only 2 out of 36 APT volumes measured on the same sample have shown the presence of NiSi: this is also in agreement with the density of precipitates measured by TEM (1 nucleus/ $\left.\mu \mathrm{m}^{2}\right)$.

\section{Discussion}

\subsection{Lateral growth kinetic}

In thin film, the lateral growth of the nuclei is the step that follows their nucleation and it takes place until a continuous layer is formed [20-27]. Before examining the nucleation of NiSi at the epitaxial $\theta$ $\mathrm{Ni}_{2} \mathrm{Si} / \mathrm{Si}$ interface, it is worth exploiting the XRD data to investigate the lateral growth of the NiSi phase. Indeed, the XRD intensity is proportional to the volume fraction of the phase and the slopes corresponding to the NiSi growth in Figure 1 and 2 are thus representative of the kinetics of the lateral growth of NiSi. The methodology used to determine the kinetics of this lateral growth will now be presented.

Coffey et al [20] have developed a kinetic model to describe the lateral growth of the nuclei taking in consideration the two-dimensional growth along the plane of the initial interface up to coalescence of the product phase. This model assumes that the nucleation barrier is low and that a fixed density of nucleation sites (n) exists at the interface. Moreover, these nuclei are chosen to have cylindrical shape with an initial thickness $\left(\mathrm{L}_{0}\right)$ and radius $\left(\mathrm{r}_{0}\right)$. Indeed, this simple shape (cylindrical particles) assumed in this model is close to the shape observed in the TEM measurements performed on this system in 
previous work [16,17]. The original Coffey's model [20] assumes that the lateral growth rate of these nuclei is limited by the reaction at the interface and can be expressed as follows:

$$
\frac{d r}{d t}=K_{L G}=K_{L G 0} \exp \left(\frac{-E_{L G}}{k_{B} T}\right)
$$

where $\mathrm{k}_{\mathrm{B}}$ is the Boltzman constant and $\mathrm{T}$ the temperature. $\mathrm{K}_{\mathrm{LG} 0}$ and $\mathrm{E}_{\mathrm{LG}}$ are the pre-exponential factor and the activation energy corresponding to the velocity of the precipitate interface.

Coffey et al [20] expressed the volume fraction of the growing phase, $\mathrm{X}_{\mathrm{V}}$, as :

$$
\frac{d X_{V}}{d t}=\frac{L}{L_{\max }} \frac{d X_{A}}{d t}+\frac{X_{A}}{L_{\max }} \frac{d L}{d t}
$$

where $X_{A}, L$, and $L_{\max }$ are the area fraction, the thickness, and the maximum thickness of the growing phase. In the initial Coffey's model, the thickening of the growing phase, $d L / d t$, is assumed to be controlled by diffusion. In order to improve the model, we considered in ref. [24] both interfacial reactions and atom diffusion through the growing NiSi layer: i.e. the Deal and Groves linear-parabolic growth law in its integral form [28].

$$
\frac{d L}{d t}=\frac{1}{L / K_{D}^{\prime}+1 / K_{R}^{\prime}} \frac{\Delta G}{k_{B} T}
$$

where $\Delta G$ is the driving force for the growth, $K_{r}$ is the average effect of reactions at both interfaces of the nuclei and $K_{t D}$ is the effective effect of both Ni and Si diffusion in NiSi layer during growth. However due to the difficulty of finding parameters related to the reaction effect on the growth of the NiSi phase at the interface and in order to limit the number of fitting parameters, the reaction term $\mathrm{K}_{\mathrm{r}}$ is neglected in the following. Moreover, as diffusion is thermally activated, the variation with 
temperature of the ratio $\Delta G / k_{B} T$ should be low compared to the exponential variation of $K_{D}^{\prime}$ and thus $\Delta G / k_{B} T$ was considered as constant. The term $K_{D}=K_{D}^{\prime} \Delta G / k_{B} T$ will thus be used in Eq.3.

As mentioned before, Fig. 1 and 2 show that for a given temperature the slope of samples that nucleate earlier is steeper than samples that nucleate later. This suggests a small difference in temperature between different experiments. In order to test this hypothesis, all the in-situ XRD measurements (11 measurements) were fitted using the Coffey's model with the assumptions described above and a single set of kinetics $\left(K_{L G 0}, E_{L G}, K_{D 0}, E_{D}\right)$ and physical $\left(L_{0}, r_{0}, n\right)$ parameters. For each experiment, the starting value of the temperature for the simulation was taken as the nominal temperature and was then slightly changed in order to match the simulated and measured XRD intensity. In the simulation, it was assumed that the lateral growth starts after the incubation time for nucleation corresponding to the time delay defined before.

As there are a relatively large number of parameters in the model, we try to fix a maximum of them: the physical parameters (volume variation, density of nuclei (n), nuclei thicknesses and the thickness of the $\theta-\mathrm{Ni}_{2} \mathrm{Si}$ at the beginning of the NiSi growth) were taken from TEM/APT experimental data and literature [16,29-31]. In particular the TEM analyses from our previous work [16] show a low density of nuclei $\left(10^{-6}\right.$ site/nm $\left.{ }^{2}\right)$ with an average thickness of about $15 \mathrm{~nm}$. The thickness of the $\theta-\mathrm{Ni}_{2} \mathrm{Si}$ at the beginning of the NiSi growth was measured to be $17 \mathrm{~nm}[16]$. The volume variation associated to the formation of $\mathrm{NiSi}$ from $\theta-\mathrm{Ni}_{2} \mathrm{Si}$ was taken as 1.24 taking into account the atomic volume of the phases and the uniform concentration of 40 at. $\% \mathrm{Si}$ in $\theta-\mathrm{Ni}_{2} \mathrm{Si}$ [32]. The activation energy for the thickening of NiSi by diffusion of the $\mathrm{Ni}$ and $\mathrm{Si}$ elements, $\mathrm{E}_{\mathrm{D}}$, was taken from reference [12] where $\mathrm{E}_{\mathrm{d}}$ was found to be $1.65 \mathrm{eV}$ but it was needed to change the pre-exponential factor, $\mathrm{K}_{\mathrm{D} 0}$, from the literature value of $2 \mathrm{~cm}^{2} / \mathrm{sec}$ [12] to a value of $15 \mathrm{~cm}^{2} / \mathrm{sec}$. This change is relatively small since the pre-exponential factor might be very sensitive to small change in the activation energy $[33,34]$. This change could be due to a smaller grain size linked to the smaller film thickness that could induce a larger contribution of grain 
boundary diffusion. However this is not really supported by the strong texture observed for NiSi (Fig. 1) that may be inherited from the epitaxial $\theta-\mathrm{Ni}_{2} \mathrm{Si}$ [35] and should lead to large grains. The smaller film thickness could also give a greater contribution of the reaction term in Eq. 3, and/or to other factors. Moreover the presence and the redistribution of Pt (Fig. 3) can also change the kinetics [36].

The experimental and simulated volume fraction of NiSi are shown in Fig. 4 for the three different isothermal temperatures $\left(200,215,230^{\circ} \mathrm{C}\right)$. The experimental XRD data were fitted with the Coffey's model and the parameters defined above. As mentioned before, the temperature for the simulation was slightly changed from the nominal value and the lateral growth was assumed to start after the incubation time for nucleation. The very good agreement between the experiments and the simulations has allowed to extract the unknown parameters $\left(K_{L G O}\right.$ and $\left.E_{L G}\right)$ involved during the lateral growth of $\mathrm{NiSi}$ at the $\theta-\mathrm{Ni}_{2} \mathrm{Si} / \mathrm{Si}$ interface:

$$
K_{L G}=2 \pm 110^{10} \exp \left(\frac{-1.75 \pm 0.05 \mathrm{eV}}{k_{B} T}\right) \mathrm{cm} / \mathrm{s} \quad \text { Eq. } 4
$$

These simulations have shown that the main part of the XRD intensity (\# to volume fraction) is mainly related to the lateral growth (up to an intensity of about $80 \%$ ) while the thickening by diffusion concerns mainly the remaining part (from 80 to $100 \%$ ). The determined values of the pre-exponential factor, $K_{L G O}$, and the activation energy, $E_{L G}$, for the lateral growth of the NiSi phase are thus more accurate than the ones for diffusion parameters $\left(K_{D O}, E_{D}\right)$. Moreover, changes in the diffusion parameters are not strongly influenced by the values of the $K_{L G 0}, E_{L G}$. The value of $K_{L G 0}$ and $E_{L G}$ appears to be relatively large. Although a large value of the pre-exponential factor could be related to a large value of the activation energy $[33,34]$, it is interesting to compare them to other cases where lateral growth has been measured. The Coffey's model was first developed to interpret differential scanning calorimetry (DSC) measurements performed on free-standing multilayers of $\mathrm{Nb} / \mathrm{Al}$ or $\mathrm{Ni} / \mathrm{a}-$ $\mathrm{Si}$ [20]: it evidenced the nucleation and lateral growth of, respectively, the $\delta-\mathrm{Ni}_{2} \mathrm{Si}$ and $\mathrm{NbAl}_{3}$ in these multilayers and has-allowed determining the product of the nucleation site density by the lateral growth 
velocity $\left(n K_{L G 0}^{2}\right.$ ) for each of these phases. Note that only the product of these two parameters can be determined with the Coffey's model and DSC measurements but not each parameter individually. The pre-exponential factor in Eq. 4 is thus dependent on the nucleation density. The modified model considering nucleation and lateral growth as well as normal growth controlled by diffusion and interface reaction was more recently used to determine the velocities characterizing the lateral growth of $\delta-\mathrm{Ni}_{2} \mathrm{Si}$ and $\mathrm{Pd}_{2} \mathrm{Si}$ from DSC measurement [24]. The parameters determined used-in these different works are reported in Tab. 1.
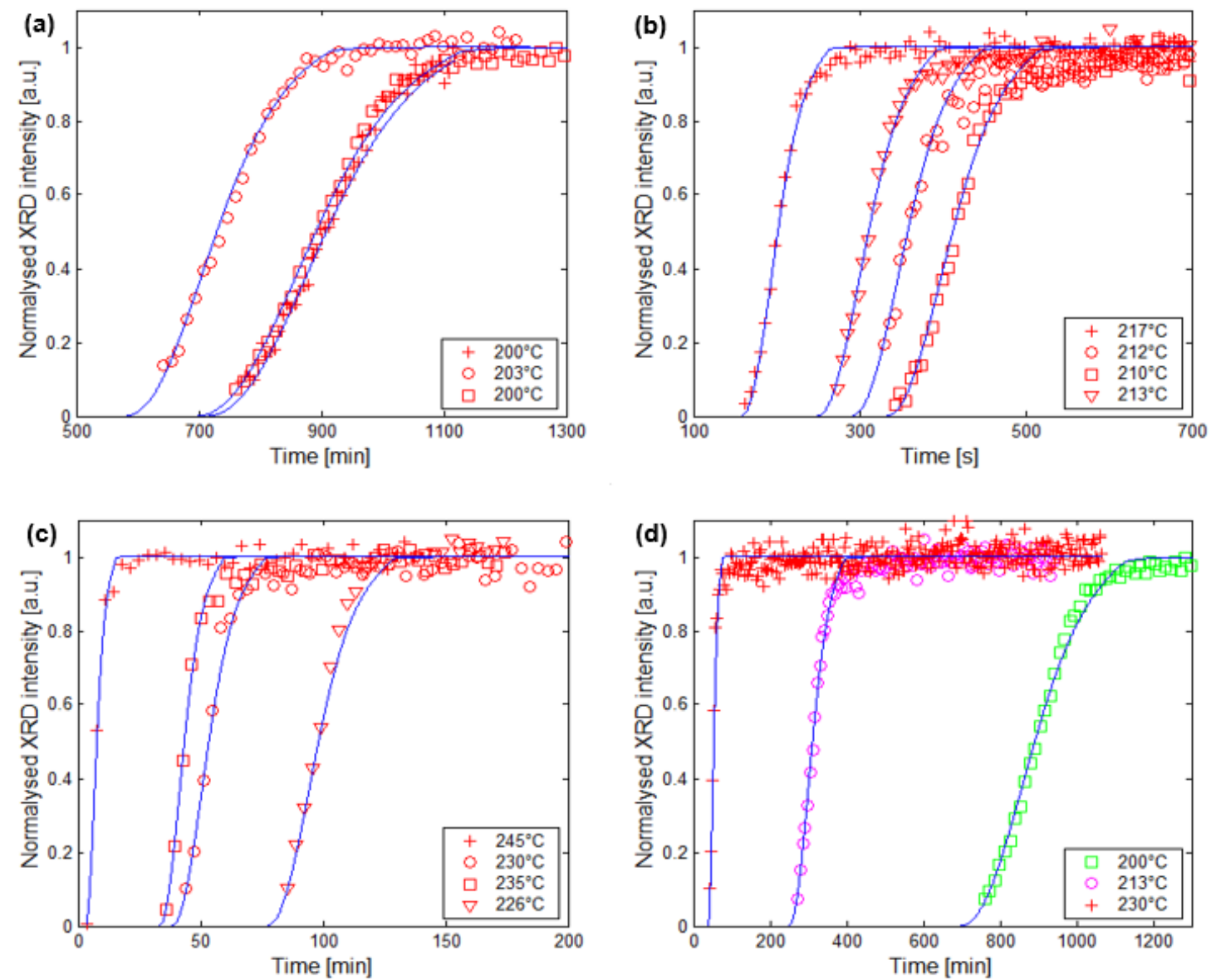

Figure 4: Simulation using the modified Coffey's model of the experimental XRD intensity that are proportional to the volume fraction of the NiSi phase. a) Experiments performed at the nominal temperature $\mathrm{T}_{\text {nom }}=200^{\circ} \mathrm{C} \mathrm{b}$ ) experiments performed at $\mathrm{T}_{\text {nom }}=215^{\circ} \mathrm{C} \mathrm{c}$ ) experiments performed at $\mathrm{T}_{\text {nom }}=230^{\circ} \mathrm{C} \mathrm{d}$ ) comparison of the different temperatures. The experiments are indicated by symbols and the simulation by continuous lines. The temperature indicated in the legend are the temperature used for the simulation 
Figure 5.a compares the lateral growth velocity obtained for $\mathrm{NiSi}$ to the ones obtained for $\delta-\mathrm{Ni}_{2} \mathrm{Si}$ and $\mathrm{Pd}_{2} \mathrm{Si}$ [24]. The velocities have similar values in the temperature range typical of the silicide growth in thin film. However, the variation with temperature is larger for the velocity of NiSi which is lower at low temperature than the one for $\delta-\mathrm{Ni}_{2} \mathrm{Si}$. This may explain partly why $\delta-\mathrm{Ni}_{2} \mathrm{Si}$ is formed before NiSi during thin film reaction since the lateral growth will be slower for NiSi. Unfortunately, there is no data available for the lateral growth of $\theta-\mathrm{Ni}_{2} \mathrm{Si}$ the first phase that forms in the $\mathrm{Ni}(10 \mathrm{at} \% \mathrm{Pt}) / \mathrm{Si}$ system. This data could allow a better understanding of the reaction between $\mathrm{Ni}(10 \mathrm{at} \% \mathrm{Pt})$ thin film and the Si substrate.

\begin{tabular}{|c|c|c|c|c|c|c|c|}
\hline System & Phase & $\mathrm{L}_{0}(\mathrm{~nm})$ & $\mathrm{n}\left(\mathrm{sites} / \mathrm{nm}^{2}\right)$ & $\mathrm{K}_{\mathrm{LG} 0}(\mathrm{~cm} / \mathrm{s})$ & $n K_{L G 0}^{2}\left(\mathrm{~s}^{-2}\right)$ & $\mathrm{E}_{\mathrm{LG}}(\mathrm{eV})$ & Ref. \\
\hline $\mathrm{Ni} / \mathrm{Si}$ & $\mathrm{Ni}_{2} \mathrm{Si}$ & 10 & - & - & $10^{28}$ & 1.5 & {$[20]$} \\
\hline $\mathrm{Nb} / \mathrm{Al}$ & $\mathrm{Ni}_{2} \mathrm{Si}$ & 30 & - & - & $310^{20}$ & 1.5 & {$[20]$} \\
\hline $\mathrm{Pd} / \mathrm{Si}$ & $\mathrm{Pd} 2 \mathrm{Si}$ & 5 & $500^{*} 10^{-6}$ & 15 & $110^{13}$ & 0.85 & {$[24]$} \\
\hline $\mathrm{Ni} / \mathrm{Si}$ & $\mathrm{Ni}_{2} \mathrm{Si}$ & 7 & $30^{*} 10^{-6}$ & 13 & $510^{11}$ & 0.85 & {$[24]$} \\
\hline $\mathrm{Ni}(10$ at.\% Pt $) / \mathrm{Si}$ & $\mathrm{NiSi}$ & $15 \pm 2$ & $1 \pm 0.5^{*} 10^{-6}$ & $2 \pm 110^{20}$ & $4 \pm 210^{28}$ & $1.75 \pm 0.5$ & This work \\
\hline
\end{tabular}

Table 1: Values of the parameters for the lateral growth obtained in this study compared to the values obtained in literature for $\mathrm{Pd}_{2} \mathrm{Si}, \mathrm{Ni}_{2} \mathrm{Si}$ and $\mathrm{NbAl}_{3}[20,24]$. In ref [20], only the product $n K_{L G 0}^{2}$ is given.

To compare the lateral growth of these different phases, it may be more relevant to compare the volume of phase formed by lateral growth which is proportional to the product of nucleation site density (density of growing precipitates) by the square of the lateral growth velocity (proportional to the surface of the precipitates). Fig. 5.b. shows that there are several orders of magnitude between these products for the different phases. Fig. 5 also emphasizes that, at low temperature, not only the velocity but also the density of nucleation sites are lower for $\mathrm{NiSi}$ compared to $\delta-\mathrm{Ni}_{2} \mathrm{Si}$. This low nucleation site density could be indicative of a difficulty of nucleation that should be at the origin of the time (or 
temperature) delay between the $\theta-\mathrm{Ni}_{2} \mathrm{Si}$ formation and the $\mathrm{NiSi}$ formation. It could be linked also to the microstructure of the $\theta-\mathrm{Ni}_{2} \mathrm{Si}$ phase since nucleation is expected to occur at triple junction or quadruple point (intersection between the substrate and a triple junction of three grains). Whatsoever, the values obtained in our study (Tab. 1 and Fig. 5) should be more reliable since the morphological parameters were obtained by TEM [16]. The difference between the parameters may be linked to different techniques (DSC versus XRD), to different samples (multilayer versus thin film), and to different intrinsic properties of the intermetallics.
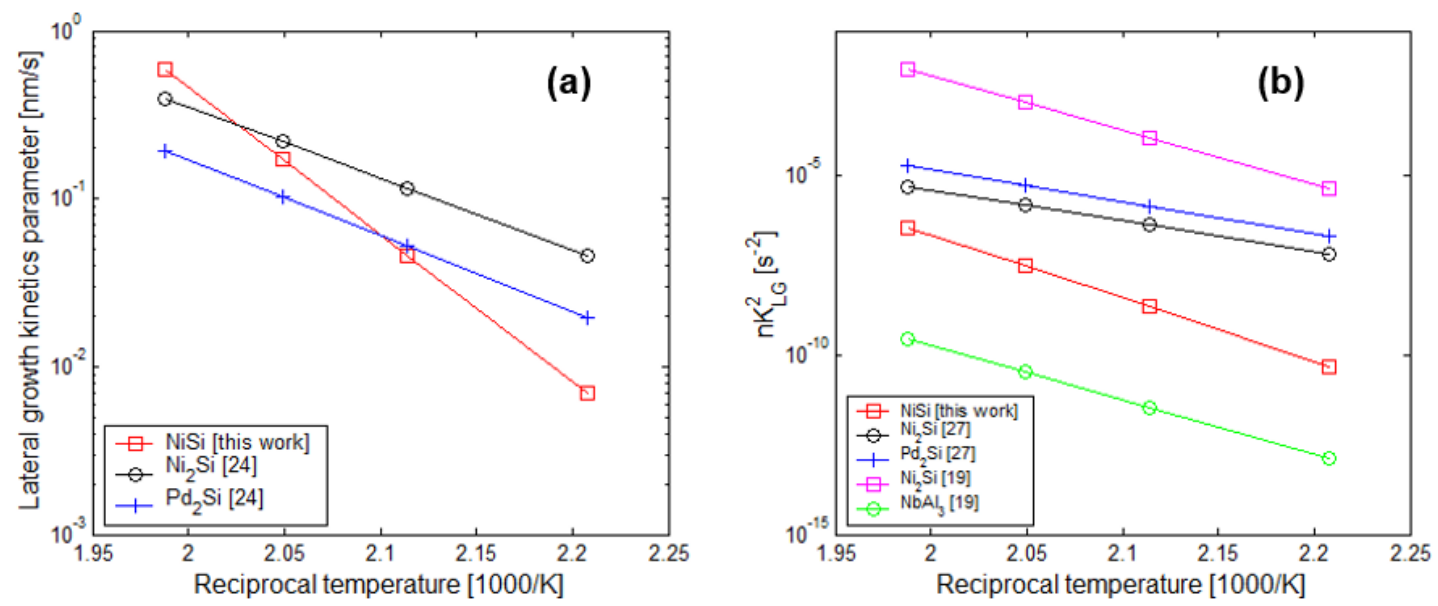

Figure 5: Arrhenius representation of the kinetics parameters for the lateral growth a) Velocity for silicide b) Product of nucleation site density by the lateral growth velocity for NiSi compared the other intermetallic compounds [20,24].

The good accordance between the experiments and the simulation could indicate that the NiSi lateral growth is well described by the Coffey's model and that its kinetics is limited by the interface mobility (Eq. 1). However the Coffey's model is very simple and more complex models [16,17,37-40] have been developed for the lateral growth but they require several parameters (interface energies, diffusion along interfaces) that are not known and/or very difficult to measure [16,17,37-40].

The NiSi lateral growth kinetics appears to be faster than the normal growth that is usually limited by both diffusion and interface reaction (Eq. 3). This is the main explanation for the high aspect ratio 
between the height and the length of the precipitates because the precipitates do not have the time to thicken during their lateral growth.

More precisely, the thickness of the precipitate during lateral growth should depend on the inverse of the lateral growth velocity $[16,17,37-40]$ : i.e. the larger the velocity the lower the characteristic thickness for lateral growth. Moreover this velocity is proportional to the driving force for the formation of phase while the thickness is inversely proportional to this driving force [37]. Since the driving force for the second phase (NiSi) should be lower than the driving force for the first phase $(\theta$ $\mathrm{Ni}_{2} \mathrm{Si}$ or $\delta-\mathrm{Ni}_{2} \mathrm{Si}$ ) [13], the velocity is expected to be lower and the thickness higher for $\mathrm{NiSi}$ than for the other silicides (Tab. 1). Moreover, a lower driving force should lead to a more difficult nucleation and an increased incubation time as will be discussed next. A difficult nucleation means also a lower nucleation site density: a lower nucleation site density gives more time for the precipitate lateral growth before impingement occurs and thus increases the aspect ratio of these precipitates.

The lateral growth ends up when the growing phase (NiSi) forms a continuous layer since a continuous layer can only grow by normal growth. Thus the lateral growth concerns only the limited part of the layer, i.e. a slab with a thickness about equal to the characteristic thickness for lateral growth (around $10 \mathrm{~nm}$ ), while the growth above this thickness occurs by normal growth. For large film thicknesses, the normal growth will control the growth over a large proportion of the growing phase. Conversely, for small film thickness (i.e. in the rage of the characteristic thickness for lateral growth), a large part of the layer will be formed by lateral growth. In our study, the film thickness $(10 \mathrm{~nm}$ of metal $\sim 17 \mathrm{~nm}$ of $\theta-\mathrm{Ni}_{2} \mathrm{Si} / \mathrm{Si}$ ) is about the $\mathrm{NiSi}$ precipitate thickness during lateral growth (around $15 \mathrm{~nm}$ ). Most of the NiSi volume (about 70\%) is thus formed by lateral growth since only a few nm of $\theta-\mathrm{Ni}_{2} \mathrm{Si}$ is left after this growth step. More generally, for a given thickness of metal, the volume fraction of new phase due to lateral growth will be higher for lower velocity and/or lower driving force. 


\subsection{Nucleation kinetic}

The time delays observed before the formation of NiSi could correspond either to an incubation time for the $\mathrm{NiSi}$ nucleation at the $\theta-\mathrm{Ni}_{2} \mathrm{Si} / \mathrm{Si}(100)$ interface or to the stress relaxation of $\theta-\mathrm{Ni}_{2} \mathrm{Si}$. Mangelinck et al [15] observed a time delay between the formation of NiSi and the end of the growth of $\delta-\mathrm{Ni}_{2} \mathrm{Si}$ that follows an Arrhenius behaviour with activation energy around 1.2 and $1.7 \mathrm{eV}$ for pure $\mathrm{Ni}$ and $\mathrm{Ni}(5$ at.\% $\mathrm{Pt})$ respectively. This time delay was related to the strain relaxation in the $\delta-\mathrm{Ni}_{2} \mathrm{Si}$ phase [15]. Indeed, even if the stress/strain behaviour is complex during the silicide growth [41], the stress development due to volume change is concomitant with the stress relaxation during the growth while only stress relaxation takes place when the growth is finished by full consumption of the metal [42]. This last relaxation was found to be correlated to the time delay: i. e. it was found that the growth of NiSi occurs only when the stress relaxation in the $\delta-\mathrm{Ni}_{2} \mathrm{Si}$ is completed [15]. A similar behavior might be at the origin of the time delay between $\theta-\mathrm{Ni}_{2} \mathrm{Si}$ and $\mathrm{NiSi}$. However, $\theta-\mathrm{Ni}_{2} \mathrm{Si}$ phase is not easily detectable with XRD and it is thus difficult to measure its stress relaxation.

Previous studies $[12,43]$ have shown that NiSi has no difficulties to nucleate at the $\delta-\mathrm{Ni}_{2} \mathrm{Si} / \mathrm{Si}$ interface and that its growth is controlled by $\mathrm{Ni}$ diffusion. However, for $\mathrm{Ni}(10 \% \mathrm{Pt})$, the NiSi growth occurs at the epitaxial $\theta-\mathrm{Ni}_{2} \mathrm{Si} / \mathrm{Si}$ interface. This interface being epitaxial, its energy should be low and render the nucleation of new phases more difficult. This is in accordance with the observation of NiSi precipitates. Indeed, our APT analyses and previous TEM measurements [16,17] performed on the same samples, show a low density of NiSi nuclei at the $\theta-\mathrm{Ni}_{2} \mathrm{Si} / \mathrm{Ni}$ interface instead of the formation of a complete layer of $\mathrm{NiSi}$ during its growth. This low density of NiSi nuclei distributed at the interface suggests a difficulty in the NiSi nucleation.

To better explain these observations, we will now consider all the results gathered on the NiSi nucleation at the $\theta-\mathrm{Ni}_{2} \mathrm{Si} / \mathrm{Si}$ interface and compare it to the one at the $\delta-\mathrm{Ni}_{2} \mathrm{Si} / \mathrm{Si}$ interface. We first recall the equation from the classical nucleation theory that states the direct dependence of the 
nucleation barrier $\left(\Delta \mathrm{G}^{*}\right)$ on the variation of the interfaces energies $(\Delta \sigma)$ and the change in volume energy - driving force - $(\Delta \mathrm{G})$ of the newly formed phase.

$$
\Delta G^{*} \propto \frac{(\Delta \sigma)^{3}}{(\Delta G)^{2}}
$$

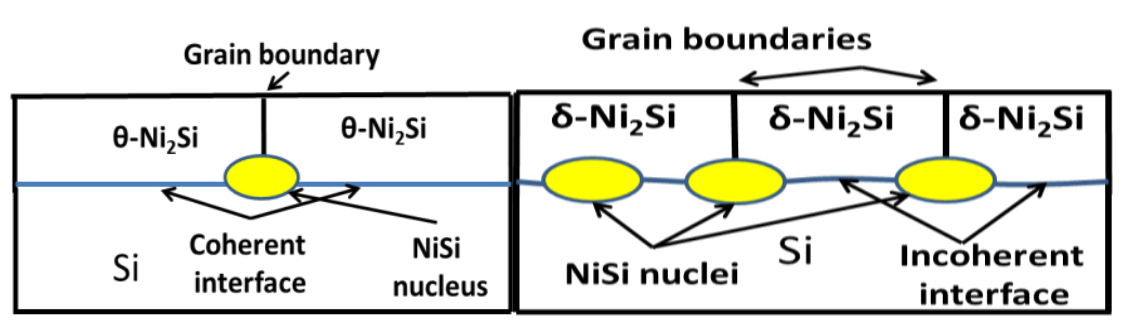

Figure 6: Schematic of $\mathrm{NiSi}$ nucleation at the expense of $\theta-\mathrm{Ni}_{2} \mathrm{Si}$ or of $\delta-\mathrm{Ni}_{2} \mathrm{Si}$ phase.

For a general case of NiSi nucleation at the first phase (FP)/Si interface, we can write:

$$
\Delta G^{*} \propto \frac{\left(\sigma_{N i S i / S i}+a_{1} \sigma_{N i S i / F P}-a_{2} \sigma_{F P / S i}-a_{3} \sigma_{G B}\right)^{3}}{\left(G_{N i S i}-\frac{1-x_{N i S i}}{1-x_{F P}} G_{F P}\right)^{2}}
$$

where $\sigma \mathrm{NiSi} / \mathrm{Si}$ and $\sigma \mathrm{NiSi} / \mathrm{FP}$ are the energies of the newly created interfaces $\mathrm{NiSi} / \mathrm{Si}$ and $\mathrm{NiSi} / \mathrm{FP}$ respectively and $\sigma_{F P / S i}$ and $\sigma_{G B}$ are the energies of the vanishing interface between FP and Si and the grain boundaries energy in FP phase respectively. $G_{N i S i}$ and $G_{F P}$ are the volume energy of NiSi and FP respectively.

It has been shown that the driving force $(\Delta \mathrm{G})$ for the NiSi formation from $\theta-\mathrm{Ni}_{2} \mathrm{Si}$ or $\delta-\mathrm{Ni}_{2} \mathrm{Si}$ phases in contact with silicon are similar [44]. Moreover, the coherent nature of the interface between $\theta-\mathrm{Ni}_{2} \mathrm{Si}$ and the Si substrate with the epitaxial relationship [10] implies a low interface energy. Thus, the term $\sigma_{\mathrm{FP} / \mathrm{Si}}$ is low in the case of $\theta-\mathrm{Ni}_{2} \mathrm{Si}$ as the first phase. Consequently, this increases the nucleation barrier for $\mathrm{NiSi}$ and makes it more difficult to nucleate at the epitaxial $\theta-\mathrm{Ni}_{2} \mathrm{Si} / \mathrm{Si}$ interface (Figure 6). In the 
case of $\delta-\mathrm{Ni}_{2} \mathrm{Si}$ as the first phase, the interface between the $\delta-\mathrm{Ni}_{2} \mathrm{Si}$ phase and the $\mathrm{Si}$ substrate is incoherent which means that the energy $\sigma_{\mathrm{FP} / \mathrm{Si}}$ is relatively high. High energy of the initial interface implies a decrease of the nucleation barrier for NiSi. Therefore, NiSi can nucleate more easily at the $\delta-\mathrm{Ni}_{2} \mathrm{Si} / \mathrm{Si}$ substrate interface than at the $\theta-\mathrm{Ni}_{2} \mathrm{Si} / \mathrm{Si}$ interface (Figure 6).

In addition, the presence of defects can modify the energies and since the nucleation barrier for NiSi is high at the $\theta-\mathrm{Ni}_{2} \mathrm{Si} / \mathrm{Si}$ interface, the nucleation is expected to occur on the defects that exist at the interface. The nucleation sites are thus expected to be the grain boundaries of triple lines between the $\theta-\mathrm{Ni}_{2} \mathrm{Si}$ grain boundaries and the $\theta-\mathrm{Ni}_{2} \mathrm{Si} / \mathrm{Si}$ interface or the misfit dislocations misfitat the interface. The presence of grain boundary (triple line) in $\theta-\mathrm{Ni}_{2} \mathrm{Si}$ phase should thus decrease the nucleation barrier leading to higher probability for NiSi to nucleate. However, no grain boundary was observed during TEM or APT analysis (attempts to perform EBSD were also unsuccessful due to the limited film thickness) meaning that the grain size of $\theta-\mathrm{Ni}_{2} \mathrm{Si}$ is very large in accordance with the epitaxial relationship. There should thus be only a low number of nucleation sites available. The difficulty to nucleate on a coherent interface and the low number of nucleation sites make the density of NiSi nuclei very low in the presence of $\theta-\mathrm{Ni}_{2} \mathrm{Si}$. Conversely, the grain size of the $\delta-\mathrm{Ni}_{2} \mathrm{Si}$ phase is small as shown by APT analysis over several samples in which several grain boundaries were successfully analyzed $[45,46]$. The small grain size means that a high density of grain boundaries exists allowing a lowering of the nucleation barrier for $\mathrm{NiSi}$ and a higher probability for nucleation at the expense of $\delta-\mathrm{Ni}_{2} \mathrm{Si}$. Thus, these time delays occurring before NiSi formation should be due to a difficulty in the nucleation of the NiSi phase at the $\theta-\mathrm{Ni}_{2} \mathrm{Si} / \mathrm{Si}$ epitaxial interface.

When the nucleation barrier is high enough compared to the thermal energy $k_{B} \mathrm{~T}$, there is a transient regime before the system reaches its steady state [47]. This regime is characterized by an incubation time " $t_{\text {inc }}$ " that can be written for homogeneous nucleation using different approximations as [47]:

$$
t_{i n c}=\theta_{0} \frac{1}{\pi Z^{2} \beta^{*}}=\theta_{0} \frac{A k_{B} T(\Delta \sigma)^{3}}{(\Delta G)^{4} \beta^{*}} \quad \text { Eq. } 7
$$


where $\mathrm{Z}$ is the Zeldovich factor, $\Delta G$ the change in free energy (driving force), $\Delta \sigma$ the interface free energy and $A$ a geometric factor. The factor $\theta_{0}$ depends on the chosen approximation and is close to 1 [48].

$\beta^{*}$ is the rate at which a critical cluster grows. $\beta^{*}$ can have two expressions depending on the growth limiting process: i.e. the reaction at the interface to attach the atoms on the critical cluster, or the longrange diffusion of solute atoms. Both expressions for $\beta^{*}$ contains thermally activated term (reaction at the interface or diffusion) and they can be written in the form of Arrhenius expression. If the longrange diffusion of solute atoms is the limiting process, the expression for $\beta^{*}$ is:

$$
\beta^{*}=4 \pi r^{*} \frac{x_{i}^{0}}{y_{i}^{0}} \frac{D_{i}}{\Omega}
$$

where $x_{i}^{0}$ and $y_{i}^{0}$ are the respective atomic fraction of the jumping atoms in the metastable parent phase and the stable nucleating phase. The critical radius for nucleation, $\mathrm{r}^{*}$, is proportional to $\Delta \sigma / \Delta G$. Therefore, the expression for $t_{\text {inc }}$, can be written as

$$
t_{\text {inc }}=\theta_{0} \frac{A^{\prime} k_{B} T(\Delta \sigma)^{2}}{(\Delta G)^{3} D_{i}}=t_{\text {inc } 0} \exp \left(\frac{E_{a}}{k_{B} T}\right)
$$

since $\mathrm{D}_{\mathrm{i}}$ should follow an Arrhenius behavior: $D_{i}=D_{0} \exp \left(-E_{a} / k_{B} T\right)$.

As usually assumed, $t_{\text {inc } 0}$ may be considered as independent of T the variation of $\Delta \sigma, \Delta G$ and $k_{B} T$ with $\mathrm{T}$ is usually negligible compared to the exponential term variation. The logarithm of the incubation time should thus present a linear behavior as a function of the reciprocal temperature and should increase with decreasing temperature.

The in situ XRD data allows determining the incubation time for all the experiments at different temperatures $\left(180,200,215\right.$, and $\left.230^{\circ} \mathrm{C}\right)$ and these values are reported in Tab. 2. As expected, the incubation time increases with increasing temperature. 


\begin{tabular}{|c|c|c|}
\hline $\mathrm{T}_{\text {nom }}\left[{ }^{\circ} \mathrm{C}\right]$ & $\mathrm{T}_{\text {sim }}\left[{ }^{\circ} \mathrm{C}\right]$ & $\mathrm{t}_{\text {in }}[\mathrm{s}]$ \\
\hline 180 & 180 & 360000 \\
\hline 200 & 200 & 43000 \\
\hline 200 & 203 & 35000 \\
\hline 200 & 200 & 42000 \\
\hline 215 & 217 & 9500 \\
\hline 215 & 212 & 17500 \\
\hline 215 & 210 & 20000 \\
\hline 215 & 213 & 15000 \\
\hline 230 & 245 & 180 \\
\hline 230 & 230 & 2300 \\
\hline 230 & 235 & 2000 \\
\hline 230 & 226 & 4650 \\
\hline
\end{tabular}

Table 2: Incubation time ( $\left.\mathrm{t}_{\mathrm{inc}}\right)$ obtained from the in situ XRD measurements. $\mathrm{T}_{\text {nom }}$ is the nominal temperature. $\mathrm{T}_{\text {sim }}$ is the simulated temperature used to fit the lateral growth of NiSi. The errors bars for temperature and incubation time were estimated from the fit the lateral growth to be respectively $\pm 2{ }^{\circ} \mathrm{C}$ and $\pm 1 \%$. For $\mathrm{T}_{\text {sim }}=$ $245^{\circ} \mathrm{C}$, the value of $\mathrm{t}_{\mathrm{inc}}$ is lower than the time for a XRD scan leading to a large error.

Figure 7 presents the variation of the logarithm of the incubation time as a function of the inverse of temperature. Two types of temperatures have been used in Fig. 7: either the nominal ones or the ones used for the simulation of the lateral growth. If the nominal temperature is taken into account, a large spread of the incubation time is obtained. This spread could correspond to fluctuation in nucleation that is expected in the classical nucleation theory. However if the incubation times are reported as a function of the simulated temperature, the spread becomes negligible and a linear relationship is obtained in the Arrhenius curve as expected from Eq. 9. This is a strongly suggest that the real temperature are closer to the simulated ones than to the nominal ones. Figure 7 shows thus that the variation of the logarithm of the incubation time as a function of the inverse of temperature follows a linear relationship. The activation energy for $\mathrm{NiSi}$ nucleation kinetic at the $\theta-\mathrm{Ni}_{2} \mathrm{Si} / \mathrm{Si}$ interface deduced from the fit of these data (Tab. 2) is found to be $1.9 \mathrm{eV}$ and the pre exponential factor $t_{\text {inc } 0}=3$ $10^{-16} \mathrm{~s}$, giving the following dependency: 


$$
t_{\text {inc }}=3 \pm 110^{-16} \exp \left(\frac{1.9 \pm 0.05 \mathrm{eV}}{k_{B} T}\right) s
$$

Note that a higher activation is obtained $(2.2 \pm 0.1 \mathrm{eV})$ if the nominal temperature is used instead of the temperature determined in the lateral growth simulation.

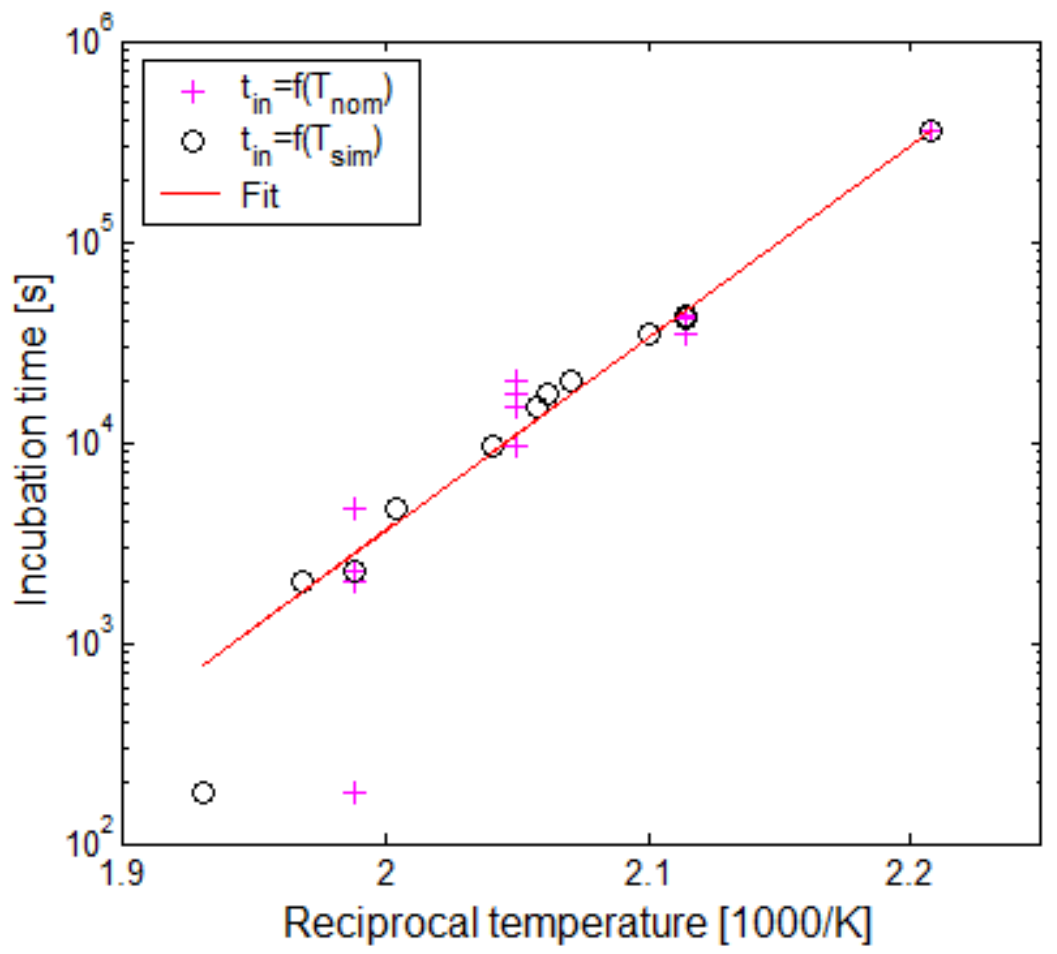

Figure 7: Incubation time as a function of the inverse of temperature. Either the temperature determined in the simulation of the lateral growth (black circles) or the nominal temperature (pink crosses) has been used. The line corresponds to the exponential fit. The value of $180 \mathrm{~s}$ for the first annealing at $230^{\circ} \mathrm{C}$ (Tab. 2) was not taken into account for the fit because of its large uncertainty.

It is worth mentioning that during the incubation time, atoms fluctuation occurs until the formation of a nucleus with a size larger than a certain threshold. This atoms fluctuation is greatly affected by temperature changes and a small change in temperature may induce an important variation in the incubation time. Therefore, the change in incubation time for a given nominal temperature should be 
due to the slight variation of temperature during the experiments that were revealed by the change in lateral growth kinetics (Fig. 4). Even a change of a few percent in the temperature can induce a large change in the incubation time (Fig. 4 and Tab. 2). This variation of the incubation time is easier to observe at low temperature since the incubation time is larger: for example, a change of $7^{\circ} \mathrm{C}$ (from 210 to $217^{\circ} \mathrm{C}$ ) can more than double the incubation time (Tab. 2 and Fig. 7). Our results show that a little variation in temperature impacts more drastically the reaction kinetics when it is controlled by nucleation than when it is controlled by diffusion (Fig. 4). This may be of great importance for the contacts in microelectronics since difference in temperatures may arise due to non-uniformity along the Si wafer during the heat treatments used to form the contacts. Furthermore, in transistors, Si is doped with different dopants and doping levels depending on the regions (source, drain, gate). These dopants can change the formation of NiSi and may have also an impact on the nucleation of NiSi $[7,49]$. Similarly the addition of Ge or C used to modify the carrier mobility of the Si semiconductor may affect the nucleation. As nucleation is expected to occur at defects such as triple line or junction between triple line and $\mathrm{Si}$ interface, it could also depend on the local microstructure (grain size, texture...).

However one should emphasize that our measurements were done at low temperature compared to the heat treatments performed to fabricate the contacts through the microelectronics processes. Indeed rapid thermal annealing (RTA) or nanosecond laser annealing (NLA), i.e. heat treatments at high temperatures for (very) short times, are usually performed in microelectronics. Our low temperature measurements allow to reveal and measure the incubation times that are relatively large at low temperature due to the exponential variation but should be much lower at high temperatures. For example, the incubation will be less than one second accordingly with Eq. 10 at $350^{\circ} \mathrm{C}$. For RTA or NLA, the incubation time may thus not be detected and could have a lower or negligible impact.

At high temperature heat treatment such as RTA or NLA, the mechanisms associated to nucleation may also change. Indeed the driving force for nucleation may be different at high temperature: since 
the $\theta-\mathrm{Ni}_{2} \mathrm{Si}$ phase is metastable at temperature below $825^{\circ} \mathrm{C}$ in the $\mathrm{Ni}-\mathrm{Si}$ system, the driving force should be lower (and thus not in favor of the NiSi nucleation) but other factors such as the presence of Pt or the metal to Si concentration ratio could have a reverse effect on the driving force. Nucleation may also change from heterogeneous (i.e. on defects at the $\theta-\mathrm{Ni}_{2} \mathrm{Si} / \mathrm{Si}$ interface) to homogeneous (i.e. at any place of the $\theta-\mathrm{Ni}_{2} \mathrm{Si} / \mathrm{Si}$ interface) since, at high temperature, larger fluctuations may allow the higher energy configurations needed for homogeneous nucleation.

The equation developed for the nucleation incubation time (Eq. 9) is based on the classical nucleation theory for homogeneous nucleation and is only a crude estimation for the present case where nucleation occurs on defects at an interface between a silicide et Si. Nevertheless, the exponential law with an activation energy of $1.9 \mathrm{eV}$ (Eq. 10) is a good fit to the incubation times determined experimentally. This law should thus allow a better control of the contact formation in microelectronics.

In the classical nucleation theory, the incubation time is required for large enough fluctuation to overcome the nucleation barrier around nucleation site and thus to obtain nuclei with a size larger than the critical size. However the time delay for NiSi nucleation may come from some other relaxation process that could be mechanical and/or diffusive. The possible relaxation processes will be now examined.

As discussed before, the relaxation of stress was already found to play an important role in the time delay between the end of the growth of $\delta-\mathrm{Ni}_{2} \mathrm{Si}$ and the formation of $\mathrm{NiSi}$ [15]. Arrhenius behaviors with activation energy around 1.2 and $1.7 \mathrm{eV}$ were found for the time delay in case of pure $\mathrm{Ni}$ and $\mathrm{Ni}(5$ at.\% Pt) respectively [15]. The time delay was clearly related to the strain relaxation in $\delta-\mathrm{Ni}_{2} \mathrm{Si}$ since the formation of NiSi occurs only when the strain in $\delta-\mathrm{Ni}_{2} \mathrm{Si}$ is relaxed [15]. However the stress/strain behavior is complex during the silicide growth [41] [42] and no clear explanation was found for the link between strain relaxation and the formation of NiSi. Indeed if stress in the nucleating (or growing) phase (NiSi) may explain difficulty of nucleation (or growth), it is much more difficult to understand why the strain in the phase that is consumed $\left(\delta-\mathrm{Ni}_{2} \mathrm{Si}\right)$ should play a role. The activation energy for the 
time delay between $\theta-\mathrm{Ni}_{2} \mathrm{Si}$ and $\mathrm{NiSi}\left(\mathrm{E}_{\mathrm{a}}=1.9 \mathrm{eV}\right)$ is not far the one found for $\mathrm{Ni}(5$ at. $\% \mathrm{Pt})\left(\mathrm{E}_{\mathrm{a}}=1.7\right.$ $\mathrm{eV}$ ): this might suggest a similar behavior: i.e. the time delay between $\theta-\mathrm{Ni}_{2} \mathrm{Si}$ and $\mathrm{NiSi}$ might thus be related to the stress relaxation in $\theta-\mathrm{Ni}_{2} \mathrm{Si}$. However, $\theta-\mathrm{Ni}_{2} \mathrm{Si}$ phase is not easily detectable with $\mathrm{XRD}$ and it is thus difficult to measure its stress relaxation. Further investigations with more complex XRD techniques such as pole figure or 3 dimensional mapping of the reciprocal space may clarify this point. The stress relaxation in silicides is usually attributed to a creep mechanism that is related to the diffusion of the less mobile species, $\mathrm{Si}$ in the Ni silicide. The increase in activation energy between pure $\mathrm{Ni}$ and $\mathrm{Ni}(5$ at.\% Pt) might come from the role of Pt on the diffusion of Si or even to the need of the diffusion of Pt during creep. The larger activation energy found in our study $\left(\mathrm{E}_{\mathrm{a}}=1.9 \mathrm{eV}\right)$ might thus be a consequence of the higher content of $\mathrm{Pt}(10$ at.\%).

Another explanation for the time delay might be linked to the effect of Pt on the interfacial energy change. The nucleation of NiSi would be easier if the effect of Pt is to decrease the change in interface energy (Eq. 6) and thus either increase the energy of the $\theta-\mathrm{Ni}_{2} \mathrm{Si} / \mathrm{Si}$ interface or decrease the energy of the $\mathrm{NiSi} / \mathrm{Si}$ or $\theta-\mathrm{Ni}_{2} \mathrm{Si} / \mathrm{NiSi}$ interface. The redistribution of $\mathrm{Pt}$ has been studied in details in [8]: during the growth of $\theta-\mathrm{Ni}_{2} \mathrm{Si}, \mathrm{Pt}$ is incorporated in $\theta-\mathrm{Ni}_{2} \mathrm{Si}$ due to its large solubility but diffuses much less than $\mathrm{Ni}$ in $\theta-\mathrm{Ni}_{2} \mathrm{Si}[8]$. As a result, at the end of the $\theta-\mathrm{Ni}_{2} \mathrm{Si}$ growth, the $\mathrm{Pt}$ concentration is high close to the surface and decreases almost exponentially with depth in $\theta-\mathrm{Ni}_{2} \mathrm{Si}$ [8]. During the time delay, $\mathrm{Pt}$ should thus diffuse from the $\theta-\mathrm{Ni}_{2} \mathrm{Si}$ surface to the $\theta-\mathrm{Ni}_{2} \mathrm{Si} / \mathrm{Si}$ interface to reduce its own concentration gradient. One may thus expect an increasing content of $\mathrm{Pt}$ at the interface between $\theta-\mathrm{Ni}_{2} \mathrm{Si}$ and $\mathrm{Si}$ during the time delay. The Pt profile in $\theta-\mathrm{Ni}_{2} \mathrm{Si}$ when NiSi nucleates, i.e. after the time delay, (Fig. 3.d) is in agreement with the redistribution observed previously [8]. However the concentration of Pt at the $\theta-\mathrm{Ni}_{2} \mathrm{Si} / \mathrm{Si}$ interface is still very low: about $0.1 \%$ as shown in Fig 3.d). This could be due to a limited diffusion of $\mathrm{Pt}$ but it is possible that locally (especially at the intersection between the $\theta$ $\mathrm{Ni}_{2} \mathrm{Si} / \mathrm{Si}$ interface and the $\theta-\mathrm{Ni}_{2} \mathrm{Si}$ grain boundary or triple junction) the Pt concentration is higher. Whether a larger content of Pt might change the interface energies is not clear. Firstly, no strong 
segregation of Pt at the interfaces was observed (Fig. 3) that could change the energies of these

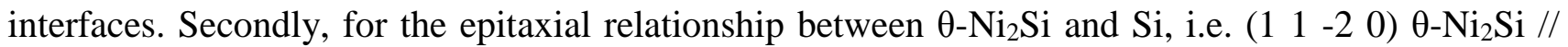
(220) $\mathrm{Si}$, the misfit is slightly negative and varies from -0.9 to $-0.1 \%$ depending on the composition of $\theta-\mathrm{Ni}_{2} \mathrm{Si}$ in the case of pure $\mathrm{Ni}$. As $\mathrm{Pt}$ is expected to increase the lattice parameter of $\theta-\mathrm{Ni}_{2} \mathrm{Si}$ because of its larger size that $\mathrm{Ni}$, a higher Pt content should decrease the misfit and thus give a better epitaxy. It would thus result in a decrease of the $\theta-\mathrm{Ni}_{2} \mathrm{Si} / \mathrm{Si}$ interface energy and thus an increase of the nucleation barrier rather than the expected decrease. Moreover to induce a change in the misfit the concentration of $\mathrm{Pt}$ at the $\theta-\mathrm{Ni}_{2} \mathrm{Si} / \mathrm{Si}$ interface should be more consequent than the $0.1 \%$ found in Fig 3.d. Note that it can be locally the case as already mentioned. The redistribution of Pt may thus not be directly at the origin of the time delay. It has also been shown that the presence of Pt change the texture of the Ni silicide [50] that is a complex combination of epitaxy, axiotaxy, as well as fiber and random components [35]. However it is not clear how the redistribution of Pt can change the texture and how this can be related to the time delay.

Another relaxation mechanism could be related to the non-stoichiometry of the $\theta-\mathrm{Ni}_{2} \mathrm{Si}$ phase. Indeed for nucleation to occur, the composition of the nucleating phase has to be reached locally [11]. The incubation time may be related to the time needed for the local composition to reach the one of NiSi. This time could be related to diffusion of $\mathrm{Ni}$, $\mathrm{Pt}$ and/or $\mathrm{Si}$ in $\theta-\mathrm{Ni}_{2} \mathrm{Si}$ and their dependency of composition of the $\theta-\mathrm{Ni}_{2} \mathrm{Si}$.

All these mechanisms and their combination could be at the origin of the time delay but it is difficult at this stage to attribute the observed time delay to one (or several) of these mechanisms or to a classical (heterogeneous or homogenous) nucleation mechanism. More experiments with advanced characterization in order to better characterize these time delay should give a better understanding. A specific point might be to determine the fluctuation of nucleation events within a given sample in comparison to the one between several samples prepared in the same way. Another important point could be to determine if the nucleation occurs at special location such as grain boundaries, triple 
lines... More fundamentally it is important to understand the nucleation at an interface between different materials. Whatsoever our results provide insights on these topics by determining how the incubation time varies with temperature. The obtained activation energy $(1.9 \mathrm{eV})$ is in agreement with the well-known semi-empirical rule saying that nucleation is suppressed for nucleation barrier higher than about $60-70 \mathrm{k}_{\mathrm{B}} \mathrm{T}$ and has reasonable rate for nucleation barrier of about $30 \mathrm{k}_{\mathrm{B}} \mathrm{T}$. Indeed, it correspond to a value of about $40 \mathrm{kT}$. Alternatively, the value of the activation energy could correspond to the relaxation mechanism detailed above. The determination of the physical meaning of $t_{i n c 0}$ and $E_{a}$ will require more investigations especially in term of simulation and modeling.

\section{Conclusion}

To conclude, several in situ XRD isothermal annealing were performed on the $10 \mathrm{~nm} \mathrm{Ni}(10$ at.\% Pt)/ $\mathrm{Si}(100)$ in order to investigate the formation of $\mathrm{NiSi}$ at the epitaxial $\theta-\mathrm{Ni}_{2} \mathrm{Si} / \mathrm{Si}$ interface. These results show an incubation time before the beginning of NiSi formation which varies with temperature. These variations appeared to be related to nucleation difficulty of the NiSi phase at the epitaxial $\theta-\mathrm{Ni}_{2} \mathrm{Si} / \mathrm{Si}$ interface with a low energy. The activation energy for NiSi nucleation at the expense of $\theta-\mathrm{Ni}_{2} \mathrm{Si}$ was estimated $1.9 \mathrm{eV}$ by plotting the variation of the incubation time as a function of the inverse of the temperature. The NiSi growth determined by in-situ XRD were fitted with the Coffey's model which allows the deduction of lateral growth kinetics $\left(E_{\mathrm{LG}}=1.75 \mathrm{eV}\right)$. This fitting suggests that after nucleation of the NiSi phase, lateral growth has a major contribution to the phase formation.

\section{Acknowledgments}

This work was supported by the CNRS-CEA "METSA" French network (FR CNRS 3507) for the TEM experiments and by the CLYM (www.clym.fr) laboratory for the access to the TEM microscopes. 
T. Epicier is greatly acknowledged for TEM measurements and M. Descoins and M. Bertoglio for technical assistance.

\section{References}

[1] R.W. Mann, L.A. Clevenger, P.D. Agnello, F.R. White, Silicides and local interconnections for high-performance VLSI applications, IBM J. Res. Dev. 39 (1995) 403-417. https://doi.org/10.1147/rd.394.0403.

[2] K.N. Tu, W.K. Chu, J.W. Mayer, Structure and growth kinetics of Ni2Si on silicon, Thin Solid Films. 25 (1975) 403-413. https://doi.org/10.1016/0040-6090(75)90058-9.

[3] C. Detavernier, A. Özcan, C. Lavoie, J.-J. Sweet, J.M.E. Harper, Kinetics of agglomeration of NiSi and NiSi2 phase formation., MRS Online Proc. Libr. Arch. 745 (2002). https://doi.org/10.1557/PROC-745-N4.12.

[4] D. Mangelinck, J.Y. Dai, J.S. Pan, S.K. Lahiri, Enhancement of thermal stability of NiSi films on (100)Si and (111)Si by Pt addition, Appl. Phys. Lett. 75 (1999) 1736-1738. https://doi.org/10.1063/1.124803.

[5] C. Lavoie, F.M. d'Heurle, C. Detavernier, C. Cabral Jr., Towards implementation of a nickel silicide process for CMOS technologies, Microelectron. Eng. 70 (2003) 144-157. https://doi.org/10.1016/S0167-9317(03)00380-0.

[6] F. Panciera, K. Hoummada, M. Gregoire, M. Juhel, N. Bicais, D. Mangelinck, Three dimensional distributions of arsenic and platinum within NiSi contact and gate of an n-type transistor, Appl. Phys. Lett. 99 (2011) 051911. https://doi.org/10.1063/1.3616150.

[7] F. Panciera, La sonde atomique tomographique : applications aux dispositifs CMOS avancés sub-45nm, (2012).

[8] F. Panciera, K. Hoummada, M. Gregoire, M. Juhel, D. Mangelinck, Pt redistribution in N-MOS transistors during Ni salicide process, Microelectron. Eng. 107 (2013) 173-177. https://doi.org/10.1016/j.mee.2013.01.029.

[9] M. Putero, L. Ehouarne, E. Ziegler, D. Mangelinck, First silicide formed by reaction of

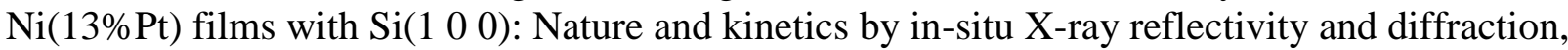
Scr. Mater. 63 (2010) 24-27. https://doi.org/10.1016/j.scriptamat.2010.02.040.

[10] F. Panciera, D. Mangelinck, K. Hoummada, M. Texier, M. Bertoglio, A. De Luca, M. Gregoire, M. Juhel, Direct epitaxial growth of $\theta-\mathrm{Ni} 2 \mathrm{Si}$ by reaction of a thin $\mathrm{Ni}(10$ at.\% Pt) film with $\mathrm{Si}(1$ 0 0) substrate, Scr. Mater. 78-79 (2014) 9-12. https://doi.org/10.1016/j.scriptamat.2014.01.010.

[11] M. El Kousseifi, K. Hoummada, M. Bertoglio, D. Mangelinck, Selection of the first Ni silicide phase by controlling the Pt incorporation in the intermixed layer, Acta Mater. 106 (2016) 193198. https://doi.org/10.1016/j.actamat.2016.01.004. 
[12] F. d'Heurle, C.S. Petersson, J.E.E. Baglin, S.J. La Placa, C.Y. Wong, Formation of thin films of NiSi: Metastable structure, diffusion mechanisms in intermetallic compounds, J. Appl. Phys. 55 (1984) 4208. https://doi.org/10.1063/1.333021.

[13] F.M. d'Heurle, Nucleation of a new phase from the interaction of two adjacent phases: Some silicides, J. Mater. Res. 3 (1988) 167-195. https://doi.org/10.1557/JMR.1988.0167.

[14] A. Alberti, C. Spinella, A. La Magna, E. Rimini, Nucleation and growth of NiSi from Ni2Si transrotational domains, Appl. Phys. Lett. 90 (2007) 053507. https://doi.org/10.1063/1.2437058.

[15] D. Mangelinck, K. Hoummada, Effect of stress on the transformation of Ni2Si into NiSi, Appl. Phys. Lett. 92 (2008) 254101. https://doi.org/10.1063/1.2949751.

[16] M. El Kousseifi, K. Hoummada, T. Epicier, D. Mangelinck, Direct observation of NiSi lateral growth at the epitaxial $\theta-\mathrm{Ni} 2 \mathrm{Si} / \mathrm{Si}\left(\begin{array}{lll}1 & 0 & 0\end{array}\right)$ interface, Acta Mater. 99 (2015) 1-6. https://doi.org/10.1016/j.actamat.2015.07.062.

[17] D. Mangelinck, M. El Kousseifi, K. Hoummada, F. Panciera, T. Epicier, Lateral growth of NiSi at the $\theta-\mathrm{Ni} 2 \mathrm{Si} / \mathrm{Si}(100)$ interface: Experiments and modelling, Microelectron. Eng. 199 (2018) 45-51. https://doi.org/10.1016/j.mee.2018.07.014.

[18] B. Gault, M.P. Moody, F.D. Geuser, A.L. Fontaine, L.T. Stephenson, D. Haley, S.P. Ringer, Spatial Resolution in Atom Probe Tomography, Microsc. Microanal. 16 (2010) 99-110. https://doi.org/10.1017/S1431927609991267.

[19] T.F. Kelly, D.J. Larson, The second revolution in atom probe tomography, Mrs Bull. 37 (2012) 150-158. https://doi.org/10.1557/mrs.2012.3.

[20] K. Coffey, L. Clevenger, K. Barmak, D. Rudman, C. Thompson, Experimental-Evidence for Nucleation During Thin-Film Reactions, Appl. Phys. Lett. 55 (1989) 852-854. https://doi.org/10.1063/1.102447.

[21] K. Coffey, K. Barmak, D. Rudman, S. Foner, Thin-Film Reaction-Kinetics of Niobium Aluminum Multilayers, J. Appl. Phys. 72 (1992) 1341-1349. https://doi.org/10.1063/1.351744.

[22] C. Bergman, E. Emeric, G. Clugnet, P. Gas, Kinetics of Reactive Diffusion in Al/Co Multilayers, Defect Diffus. Forum. 194-199 (2001) 1533-1538. https://doi.org/10.4028/www.scientific.net/DDF.194-199.1533.

[23] F. Nemouchi, D. Mangelinck, C. Bergman, P. Gas, U. Smith, Differential scanning calorimetry analysis of the linear parabolic growth of nanometric Ni silicide thin films on a Si substrate, Appl. Phys. Lett. 86 (2005) 041903. https://doi.org/10.1063/1.1852727.

[24] K. Hoummada, D. Mangelinck, A. Portavoce, Kinetic of Formation of Ni and Pd Silicides: Determination of Interfacial Mobility and Interdiffusion Coefficient by In Situ Techniques, Solid State Phenom. 172-174 (2011) 640-645. https://doi.org/10.4028/www.scientific.net/SSP.172-174.640.

[25] K. Hoummada, A. Portavoce, C. Perrin-Pellegrino, D. Mangelinck, C. Bergman, Differential scanning calorimetry measurements of kinetic factors involved in salicide process, Appl. Phys. Lett. 92 (2008) 133109. https://doi.org/10.1063/1.2905293.

[26] G. Lucadamo, K. Barmak, S. Hyun, C. Cabral Jr., C. Lavoie, Evidence of a two-stage reaction mechanism in sputter deposited $\mathrm{Nb} / \mathrm{Al}$ multilayer thin-films studied by in situ synchrotron $\mathrm{X}$ ray diffraction, Mater. Lett. 39 (1999) 268-273. https://doi.org/10.1016/S0167-577X(99)000178.

[27] R. Delattre, O. Thomas, C. Perrin-Pellegrino, C. Rivero, R. Simola, First stage of CoSi2 formation during a solid-state reaction, J. Appl. Phys. 116 (2014) 245301. https://doi.org/10.1063/1.4904852.

[28] B.E. Deal, A.S. Grove, General Relationship for the Thermal Oxidation of Silicon, J. Appl. Phys. 36 (1965) 3770. https://doi.org/10.1063/1.1713945.

[29] L.A. Clevenger, C.V. Thompson, R.C. Cammarata, K.N. Tu, Reaction kinetics of nickel/silicon multilayer films, Appl. Phys. Lett. 52 (1988) 795-797. https://doi.org/10.1063/1.99644. 
[30] J. Demeulemeester, D. Smeets, C.M. Comrie, N.P. Barradas, A. Vieira, C. Van Bockstael, C. Detavernier, K. Temst, A. Vantomme, On the growth kinetics of $\mathrm{Ni}(\mathrm{Pt})$ silicide thin films, J. Appl. Phys. 113 (2013) 163504. https://doi.org/10.1063/1.4802738.

[31] K. Hoummada, Etude de la redistribution des dopants et des éléments d'alliages lors de la formation des siliciures, Université Paul Cézanne, 2007.

[32] M. El Kousseifi, Ni silicide contacts: Diffusion and reaction in nanometric films and nanowires, Aix-Marseille University, 2014.

[33] A. Yelon, B. Movaghar, Microscopic explanation of the compensation (Meyer-Neldel) rule, Phys. Rev. Lett. 65 (1990) 618-620. https://doi.org/10.1103/PhysRevLett.65.618.

[34] J. Philibert, Some thoughts and/or questions about activation energy and pre-exponential factor, in: B.S. Bokstein, B.B. Straumal (Eds.), Diffus. Solids - Past Present Future, 2006: pp. 61-72.

[35] B. De Schutter, K. De Keyser, C. Lavoie, C. Detavernier, Texture in thin film silicides and germanides: A review, Appl. Phys. Rev. 3 (2016) 031302. https://doi.org/10.1063/1.4960122.

[36] D. Mangelinck, T. Luo, C. Girardeaux, Reactive diffusion in the presence of a diffusion barrier: Experiment and model, J. Appl. Phys. 123 (2018) 185301. https://doi.org/10.1063/1.5023578.

[37] L. Klinger, Y. Bréchet, G. Purdy, On the kinetics of interface-diffusion-controlled peritectoid reactions, Acta Mater. 46 (1998) 2617-2621. https://doi.org/10.1016/S1359-6454(97)00471-0.

[38] M. Pasichnyy, A. Gusak, Model of Lateral Growth Stage during Reactive Phase Formation, Defect Diffus. Forum. 277 (2008) 47-52. https://doi.org/10.4028/www.scientific.net/DDF.277.47.

[39] V. Vovk, G. Schmitz, R. Kirchheim, Nucleation of product phase in reactive diffusion of Al/Co, Phys. Rev. B. 69 (2004) 104102. https://doi.org/10.1103/PhysRevB.69.104102.

[40] G. Lucenko, A. Gusak, A model of the growth of intermediate phase islands in multilayers, Microelectron. Eng. 70 (2003) 529-532. https://doi.org/10.1016/S0167-9317(03)00432-5.

[41] F.M. d'Heurle, O. Thomas, Stresses during Silicide Formation: A Review, Defect Diffus. Forum. 129-130 (1996) 137-150. https://doi.org/10.4028/www.scientific.net/DDF.129130.137.

[42] S.-L. Zhang, F.M. d'Heurle, Stresses from solid state reactions: a simple model, silicides, Thin Solid Films. 213 (1992) 34-39. https://doi.org/10.1016/0040-6090(92)90471-M.

[43] T.G. Finstad, A Xe marker study of the transformation of Ni2Si to NiSi in thin films, Phys. Status Solidi A. 63 (1981) 223-228. https://doi.org/10.1002/pssa.2210630130.

[44] D. Mangelinck, K. Hoummada, I. Blum, Kinetics of a transient silicide during the reaction of Ni thin film with (100)Si, Appl. Phys. Lett. 95 (2009) 181902. https://doi.org/10.1063/1.3257732.

[45] M. El Kousseifi, K. Hoummada, D. Mangelinck, Ni silicide study at the atomic scale: Diffusing species, relaxation and grooving mechanisms, Acta Mater. 83 (2015) 488-498. https://doi.org/10.1016/j.actamat.2014.10.029.

[46] D. Mangelinck, K. Hoummada, A. Portavoce, C. Perrin, R. Daineche, M. Descoins, D.J. Larson, P.H. Clifton, Three-dimensional composition mapping of NiSi phase distribution and $\mathrm{Pt}$ diffusion via grain boundaries in Ni2Si, Scr. Mater. 62 (2010) 568-571. https://doi.org/10.1016/j.scriptamat.2009.12.044.

[47] E. Clouet, Modeling of Nucleation Processes, in: ASM Handb. Vol. 22A Fundam. Model. Met. Process., D. U. Furrer and S. L. Semiatin, 2009: pp. 203-219.

[48] K.F. Kelton, A.L. Greer, C.V. Thompson, Transient nucleation in condensed systems, J. Chem. Phys. 79 (1983) 6261-6276. https://doi.org/10.1063/1.445731.

[49] D. Mangelinck, K. Hoummada, F. Panciera, M. El Kousseifi, I. Blum, M. Descoins, M. Bertoglio, A. Portavoce, C. Perrin, M. Putero, Progress in the understanding of Ni silicide formation for advanced MOS structures, Phys. Status Solidi -Appl. Mater. Sci. 211 (2014) 152165. https://doi.org/10.1002/pssa.201300167. 
[50] C. Detavernier, C. Lavoie, Influence of Pt addition on the texture of NiSi on Si(001), Appl. Phys. Lett. 84 (2004) 3549. https://doi.org/10.1063/1.1719276. 